\title{
Safety and economic considerations of argatroban use in critically ill patients: a retrospective analysis
}

\author{
Se-Chan Kim ${ }^{*}$, Nicole Tran ${ }^{\dagger}$, Jens-Christian Schewe, Olaf Boehm, Maria Wittmann, Ingo Graeff, Andreas Hoeft \\ and Georg Baumgarten
}

\begin{abstract}
Background: Heparin-induced thrombocytopenia (HIT) causes thromboembolic complications which threaten life and limb. Heparin is administered to virtually every critically ill patient as a protective measure against thromboembolism. Argatroban is a promising alternative anticoagulant agent. However, a safe dose which still provides effective thromboembolic prophylaxis without major bleeding still needs to be identified.
\end{abstract}

Methods: Critically ill patients $(n=42)$ diagnosed with HIT at a tertiary medical center intensive care unit from 2005 to 2010 were included in this retrospective analysis. Patient records were perused for preexisting history of HIT, heparin dosage before HIT, argatroban dosage, number of transfusions required, thromboembolic complications and length of ICU stay (ICU LOS). Patients were allocated to Simplified Acute Physiology Scores above and below 30 (SAPS >30, SAPS $<30)$, respectively. For calculations, patients $(n=19)$ without previous history of HIT were compared to patients $(n=23)$ with a history of HIT before initiation of argatroban.

Results: The mean initial argatroban dosage was below $0.4 \mathrm{mcg} / \mathrm{kg} / \mathrm{min}$ regardless of SAPS score. Maintenance dosage had to be increased in patients with SAPS $<30$ to $0.54 \pm 0.248 \mathrm{mcg} / \mathrm{kg} / \mathrm{min}(p>0.05)$ to achieve effective anticoagulation. No thromboembolic complications were encountered. Argatroban had to be discontinued temporarily in 16 patients for a total of 57 times due to diagnostic or surgical procedures, supratherapeutic aPTT and bleeding without increasing the number of transfusions. A history of HIT was associated with a shorter ICU LOS and significantly reduced transfusion need when compared to patients with no history of HIT. Cost calculation favour argatroban due to increased transfusion needs during heparin administration and increase ICU LOS.

Conclusion: Argatroban can be used at doses $<0.4 \mathrm{mcg} / \mathrm{kg} / \mathrm{min}$ without an increase in transfusion requirements and at a reduced overall treatment cost compared to heparin.

Keywords: Argatroban, Heparin induced thrombocytopenia, Critical care

\section{Background}

In heparin-induced thrombocytopenia (HIT) immunoglobulin $\mathrm{G}$ is formed against multimolecular complexes of platelet factor 4 (PF4) and polyanion heparin, which potentially leads to thromboembolic life and limb threatening complications [1,2]. Clinical diagnosis is based on the $4 \mathrm{~T}$ score (Thrombocytopenia, Timing, Thrombosis, absence of other explanations) [3,4]. Typically, clinical suspicion

\footnotetext{
* Correspondence: Se-Chan.Kim@ukb.uni-bonn.de

${ }^{\dagger}$ Equal contributors

Department of Anesthesiology and Intensive Care Medicine, University Hospital Bonn, Sigmund-Freud-Str. 25, 53127 Bonn, Germany
}

of HIT is made by a platelet count drop in the 5-10 days following heparin administration. However, it should be kept in mind that in almost $60 \%$ of patients diagnosed with HIT, thrombosis occurs prior to or on the day of significant platelet decrease [5]. Differential diagnoses include EDTA-induced pseudothrombocytopenia, nonimmune heparin-associated thrombocytopenia, thrombotic-thrombocytopenic purpura, other drug-induced thrombocytopenias, disseminated intravascular coagulation, acute thrombosis-associated thrombocytopenia and sepsis [6]. Guideline recommendations consist of discontinuation of all heparin, initiation of a non-heparin anticoagulant due 
to increased risk of thromboembolic events and establishing diagnosis with a serologic assay [7]. PF4 enzyme linked immunosorbent assay (ELISA) detects immunoglobulin G antibodies and is a widely used initial assay. However, this test has poor specificity, which might lead to overdiagnosis and overtreatment of HIT, including increased risk of bleeding $[8,9]$. Furthermore, costs of alternative anticoagulants imply an additional economic burden [10]. Only a subset of anti-PF4/heparin antibodies activate platelets, which makes certain platelet activation assays, such as the heparin-induced platelet activation assay (HIPAA) and the serotonin release assay (SRA), more specific. However, HIPAA and SRA are restricted to specialized laboratories. Diagnosing HIT in critically ill patients is difficult due to the high incidence of thrombocytopenia in this patient population. The incidence varies and seems to be higher among surgical patients compared to non-surgical patients $[11,12]$.

Argatroban is a direct thrombin inhibitor and is used as an alternative anticoagulation agent in HIT. An initial dosage of $2 \mathrm{mcg} / \mathrm{kg} / \mathrm{min}$ without a bolus is recommended except for patients with heart failure, liver dysfunction, multiorgan dysfunction syndrome, severe anasarca and early post-cardiac surgery phase. A dose reduction to 0.5 $1.2 \mathrm{mcg} / \mathrm{kg} / \mathrm{min}$ is recommended in this patient population, according to the manufacturer. Argatroban follows hepatobiliary elimination. Hence it is the preferred agent in patient in renal failure. But it needs to be closely monitored and dosage must be adjusted in case of hepatic insufficiency [13]. However, the optimal dosage for critically ill patients is still under investigation [14-17]. Even recommended dosages for critically ill patients could lead to blood loss [18]. The aim of this retrospective study was to evaluate the safety of argatroban dosage in critically ill patients, operationalized as number of thromboembolic events (i.e., myocardial infarction, stroke, pulmonary embolism and peripheral arterial occlusion), increased bleeding and need for blood transfusions, as well as mortality and length of ICU stay (ICU LOS). In addition, we compared the overall costs of therapy using argatroban versus heparin.

\section{Methods}

\section{Ethics statement}

Ethical approval for this study (Ethical Committee $\mathrm{N}^{\circ}$ 061/14) was provided by the Ethical Committee Bonn University Hospital, Bonn, Germany (Chairperson Prof. K. Racké) on 26th February 2014. Informed consent was waived and data were analyzed anonymously.

\section{Patients}

This retrospective study included 42 patients (18 female/ 24 male) in a six year period (2005-2010) who were diagnosed with HIT before or during admission to the ICU and received argatroban during their stay at the surgical ICU at the University Hospital of Bonn, Germany, a tertiary care academic medical center. Initial and maintenance dosage of argatroban and transfusion requirements in these patients were evaluated to assess safe dose ranges for argatroban. Critically ill patients were allocated to Simplified Acute Physiology Score (SAPS) of above 30 (>30) and below $30(<30)$ at the time of ICU admission. Changes in SAPS score were documented daily and correlated with argatroban dose. Clinical suspicion of HIT was made if patients had a new onset of thrombocytopenia, recent heparin exposure and/or thromboembolic complications followed by PF4 ELISA. Patients with PF4 $\geq 0.4$ OD were defined as HIT positive. Confirmatory HIPAA was additionally performed. Platelet recovery following initiation of argatroban therapy served as a clinical parameter to confirm diagnosis of HIT. Patients' data and laboratory values were extracted from hospital records and the clinical information system. According to the department's policy, heparin exposure (e.g. intravenous administration) was discontinued and catheters filled with heparinized solutions were exchanged as soon as clinical suspicion of HIT was raised, while awaiting a confirmatory diagnostic testing. Initial dosage was at the discretion of the responsible physician. One patient received lepirudin before argatroban and one patient danaparoid after argatroban. A third patient received a single oral medication of $100 \mathrm{mg}$ aspirin. The argatroban dosage was adjusted for a target activated partial thromboplastin time (aPTT) 1.5 to 3 times that of the baseline aPTT. The aPTT was routinely monitored twice daily and $4 \mathrm{hrs}$ after dosing changes per ICU policy. Bleeding was assessed by hemoglobin fall and transfusion monitoring of packed red blood cells (PRBC), fresh frozen plasma (FFP) and platelets (i.e., $\geqq 2$ PRBC in 24 hours and/or a fall in hemoglobin $\geqq 2 \mathrm{~g} / \mathrm{dL}$ ). Recognition and diagnosis of thromboembolic complications (e.g., myocardial infarction, stroke, pulmonary embolism and peripheral arterial occlusion) were included in the daily intensive care routine. Renal function was evaluated with serum creatinine, blood urea nitrogen $(B U N)$ and creatinine clearance. Acute kidney failure was treated with continuous renal replacement therapy (CRRT). Liver function tests included bilirubin, liver enzymes (alanine/aspartate aminotransferase (ALT/AST), gamma-glutamyl transferase (GT)) and albumin. Moderate elevation of serum aminotransferase was defined by more than 3 times the upper limit of normal [19]. Respiratory failure was defined as $\mathrm{PaO} 2 / \mathrm{FiO} 2<200 \mathrm{mmHg}$ [20]. Furthermore we compared ICU LOS and transfusion requirements for patients with a history of HIT with those patients who received heparin shortly before HIT was suspected (no history of HIT) and argatroban was initiated. The results were used for cost calculations of argatroban versus heparin administration. Calculations were based on current 
wholesale price of 2.17 EUR per 25.000 IE heparin and 188.12 EUR for $250 \mathrm{mg}$ argatroban. The investigated parameters comprised total daily anticoagulant administration, duration of heparin administration before initiation of argatroban, blood transfusion and ICU LOS were incorporated in the total cost calculation.

\section{Statistical analysis}

All data are reported in mean \pm standard error of the mean (SEM). A Student $\mathrm{t}$ - test or a Mann-Whitney $U$ test was performed where appropriate. Significant differences were considered to exist at $p<0.05$.

\section{Results}

Patients' demographics are summarized in Table 1.

\section{Diagnosis of HIT}

Argatroban was initiated in all patients with a history of HIT and suspected HIT which was clinically diagnosed by a history of heparin exposure and thrombocytopenia based on the $4 \mathrm{~T}$ score $[3,4]$. Anticoagulation was initiated when there was no clinical sign of perioperative bleeding and aPTT was in the lower reference range. The average time elapsed from time of ICU admission to starting anticoagulation was $13.22 \pm 4.24$ hours for patients with a history of HIT and $8.25 \pm 3.28$ hours in patients without a history of HIT $(p>0.05)$. While 23 (54.8\%) patients had a history of HIT, 19 patients (45.2\%) with no history of HIT had received heparin for $7.5 \pm 1.3$ days before HIT was suspected. Exposure to heparin was found in 17 (85\%) patients (906.4 \pm $186.5 \mathrm{IE} / \mathrm{h}$ ) up to the day before initiation of argatroban. In the remaining 2 patients, heparin infusion was interrupted more than 24 hours before initiation of

\begin{tabular}{ll}
$\begin{array}{l}\text { Table } 1 \text { Demographic data (mean } \pm \text { SEM, } \boldsymbol{n}=\mathbf{4 2} \text {, except } \\
\text { when marked otherwise) }\end{array}$ \\
\hline Age (years) & $61 \pm 2.4$ \\
Body weight (kg) & $84.4 \pm 3.2$ \\
Body Mass Index kg/m² & $29.2 \pm 1.2$ \\
Length of ICU-Stay (days) & $15 \pm 2.9$ \\
history of HIT & $6.0 \pm 1.2, n=23$ \\
no history of HIT & $25.8 \pm 5.0, n=19$ \\
SAPS <30 & $11 \pm 3.0, n=16$ \\
SAPS >30 & $18 \pm 4.2, n=26$ \\
SAPS score on ICU admission & $34 \pm 2.2$ \\
Sepsis & $14(33 \%)$ \\
Renal replacement therapy & $17(40.5 \%)$ \\
Respiratory failure & $31(73.8 \%)$ \\
Veno-arterial ECMO & $1(2.4 \%)$ \\
Death* & $6(14.2 \%)$
\end{tabular}

*Death was unrelated to bleeding, thromboembolic complication or argatroban. argatroban. Based on the $4 \mathrm{~T}$ score, there was a high probability $(4 \mathrm{~T}=6-8)$ of HIT in $4(21.1 \%)$ patients with no history of HIT, intermediate $(4 \mathrm{~T}=4-5)$ in $10(52.6 \%)$ and low probability $(4 \mathrm{~T} \leqq 3)$ in 5 patients $(26.3 \%)$. In patients with no history of HIT, PF4 ELISA was positive in 17 cases $(94.4 \%)$ out of 18 tested patients. In all 5 patients with low HIT probability score, PF4 ELISA and HIPAA were positive, respectively. Mean platelet count was $148.6 \pm 19.58 \mathrm{G} / \mathrm{L}$ at time of ICU admission.

\section{Bleeding and thromboembolic complications}

The initial dosage of argatroban anticoagulation was $0.37 \pm 0.069 \mathrm{mcg} / \mathrm{kg} / \mathrm{min}$ in SAPS $>30$ and $0.35 \pm 0.103$ $\mathrm{mcg} / \mathrm{kg} / \mathrm{min}$ in SAPS $<30$ (Figure 1). Maintenance dosage was slightly reduced in SAPS $>30$ to $0.32 \pm 0.067$ $\mathrm{mcg} / \mathrm{kg} / \mathrm{min}$ to achieve aPTT-prolongation 1.5 to 3 times of the baseline. In SAPS $<30$, maintenance dosage was increased to $0.54 \pm 0.248 \mathrm{mcg} / \mathrm{kg} / \mathrm{min}$ to achieve effective anticoagulation (Figure $1, p>0.05$ ). The initial dosage in our study was below the manufacturer's recommendations for critically ill patients with organ failure and patients with cardiac surgery $(0.5-1.2 \mathrm{mcg} / \mathrm{kg} / \mathrm{min})$. There was no correlation between initial dosage $(r=$ - 0.073) or maintenance dosage adjustment $(r=-0.326)$ and change in SAPS scores. Duration of argatroban therapy was $8.4 \pm 2.11$ days. Effective anticoagulation was monitored with aPTT which was prolonged 1.25 -fold by $8.38 \pm 2.0 \mathrm{~s}$ as compared with a mean aPTT prior to argatroban $(45.12 \pm 2.00 \mathrm{~s}, n=41$ vs. $36.02 \pm 1.52 \mathrm{~s}, n=32$; $p=<0.001)$. According to local hospital guidelines, anticoagulation for thromboembolic prophylaxis was efficient with an aPTT in the range of 35 to $40 \mathrm{~s}$. In ten patients with a history of HIT, a baseline aPTT was not available before initiation of argatroban anticoagulation. None of the study patients developed a newly diagnosed

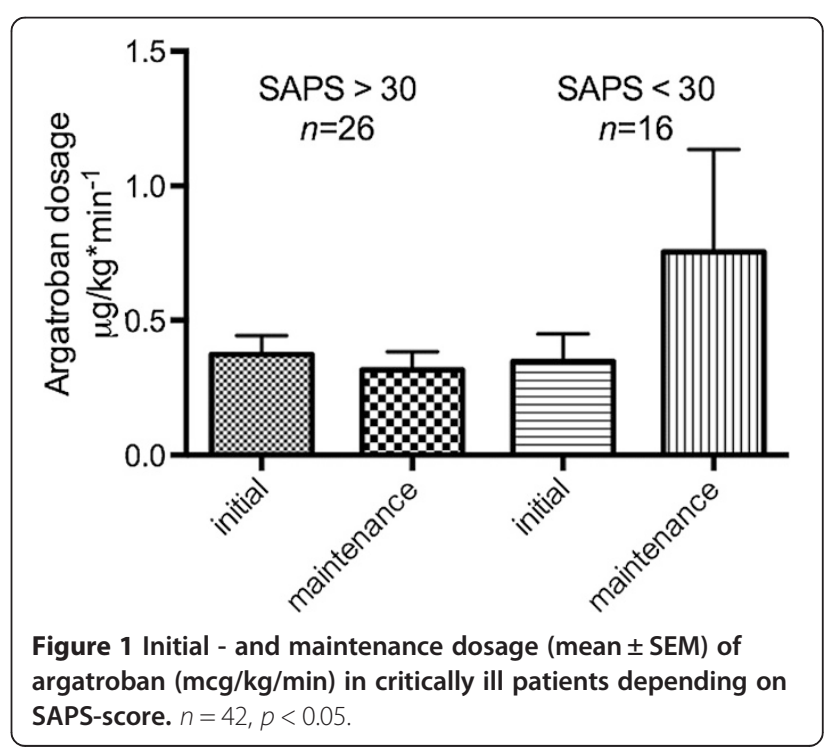


thromboembolic complication under argatroban. Major bleeding was defined by a hemoglobin fall $\geqq 2 \mathrm{~g} / \mathrm{dL}$ and a transfusion of $\geqq 2$ PRBCs within $24 \mathrm{~h}$. Argatroban infusion was discontinued temporarily in 16 patients for a total of 57 times. Reasons for discontinuation included diagnostic or surgical procedures $(20 / 57 ; 35.1 \%)$, supratherapeutic aPTT $(14 / 57 ; 24.6 \%)$ and bleeding $(12 / 57 ; 21.1 \%)$. Hence, with this management and monitoring, red blood cell transfusion under argatroban therapy was not increased (Figure $2 \mathrm{~A}$ and $2 \mathrm{~B}, p>0.05$ ). Transfusions of fresh frozen plasma and platelets were not significantly affected by argatroban therapy.

\section{Elevated serum aminotransferases and argatroban}

Moderate elevated serum aminotransferases ( $>3$ times of the upper limit of normal) and increased total bilirubin were found in seven of the investigated patients. Initial dosage in these patients was slightly higher $(0.40 \pm 0.14$ $\mathrm{mcg} / \mathrm{kg} / \mathrm{min}, n=7)$ than in patients with normal hepatic parameters $(0.36 \pm 0.06 \mathrm{mcg} / \mathrm{kg} / \mathrm{min}, n=35 ; p=0.7752)$. However, the initial dosage was slightly below the recommendation of $0.5 \mathrm{mcg} / \mathrm{kg} / \mathrm{min}$ in patients with liver dysfunction (Child Pugh Class B). There was no difference in maintenance dosage during the rest of the ICU stay $(0.42 \pm 0.09 \mathrm{mcg} / \mathrm{kg} / \mathrm{min}, n=7$ vs. $0.40 \pm 0.12 \mathrm{mcg} /$ $\mathrm{kg} / \mathrm{min}, n=35 ; p=0.9490$ ). In three patients with moderate elevated aminotransferases, argatroban therapy had to be discontinued due to hemoglobin fall or supratherapeutic aPTT. In these patients duration of discontinuation was disproportionately higher: $63.3 \%$ of the total interruption time was due to a fall in hemoglobin $>2 \mathrm{~g} / \mathrm{dL} ; 49.1 \%$ of total time of interruption in all patients due to supratherapeutic aPTT. Although, overall transfusion of blood components was unaffected by argatroban therapy in patients with moderate elevated aminotransferases, this data suggests a higher bleeding tendency in patients with moderate elevated aminotransferases.

\section{Impact of history of HIT}

There was a significant shorter length of stay in the ICU for patients with a history of HIT $(6.0 \pm 1.2$ days, $n=23)$ compared to patients with no history of HIT (25.8 \pm 5.0 days, $n=19 ; p<0.001$, Table 1 , Figure 3A). Patients with no history of HIT had a slightly but not significantly higher SAPS-score $(30.8 \pm 2.7, n=23$ vs. $37.3 \pm 3.5, n=19$; $p=0.1459$, Figure $3 \mathrm{~B})$. ICU LOS was $7.5 \pm 1.3$ days before HIT diagnosis was established. Patients with a history of HIT received argatroban $13.22 \pm 4.24$ hours after admission to the ICU. Red blood cell transfusion was significantly less in patients with a history of HIT $(4.4 \pm 2.8$ PRBC) before initiation of argatroban when compared with transfusion need of patients with no history of HIT $(9.9 \pm 3.6$ PRBC) (Figure $4, p<0.05)$. For FFP no statistical difference was found (Figure 5). Platelet transfusion was significantly lower during argatroban therapy in patients with a history of HIT $(0.2 \pm 0.1$ platelet units) than in patients with no history of HIT ( $2.3 \pm 1.3$ platelet units) (Figure $6, p<0.05)$.

\section{Cost calculations}

Patients $(n=19)$ receiving heparin before HIT was suspected served as a comparison group for cost calculation of argatroban versus heparin administration. In this study population the mean dosage for heparin was 906.4 \pm 186.5 IE/h or $21744 \mathrm{IE} / \mathrm{d}(n=19)$, whereas the maintenance dosage of argatroban was $0.41 \pm 0.10 \mathrm{mcg} / \mathrm{kg} / \mathrm{min}$ $(n=42)$ or $49.6 \mathrm{mg} / \mathrm{d}(0.41 \mathrm{mcg} \times 84 \mathrm{~kg} \times 1440 \mathrm{~min})$.

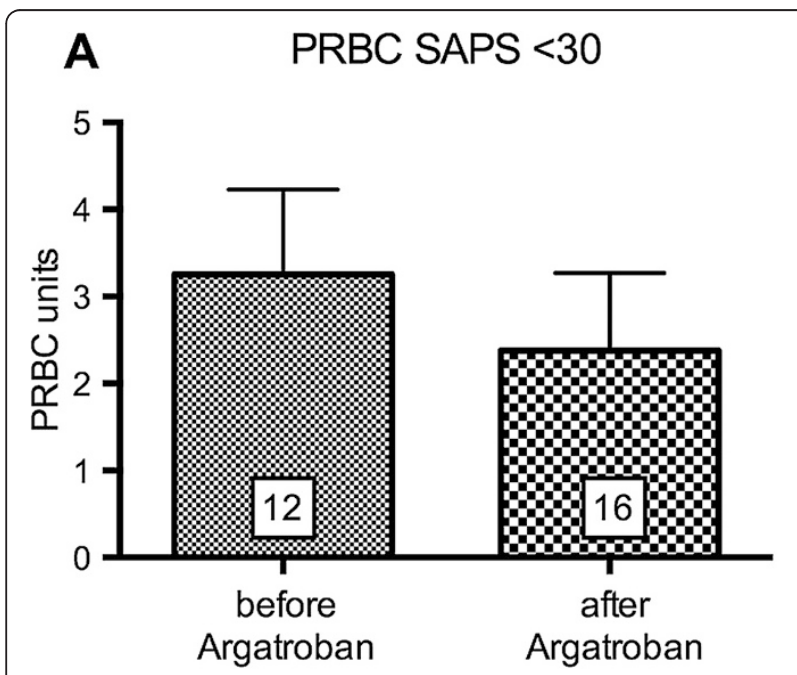

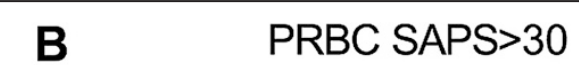

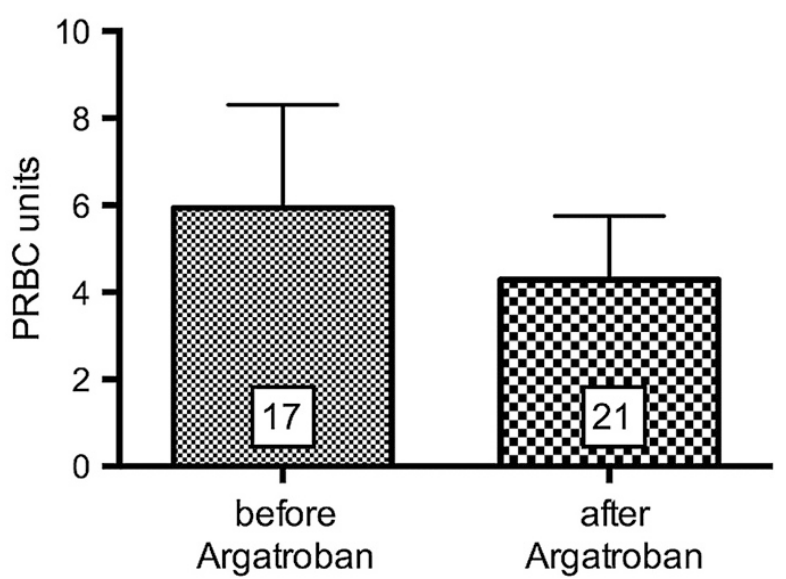

Figure 2 Red blood cell transfusion under argatroban. PRBC transfusion (mean \pm SEM) in patients with (A) SAPS $<30$ and (B) SAPS $>30$ before and during argatroban therapy during ICU stay. $p<0.05$. 


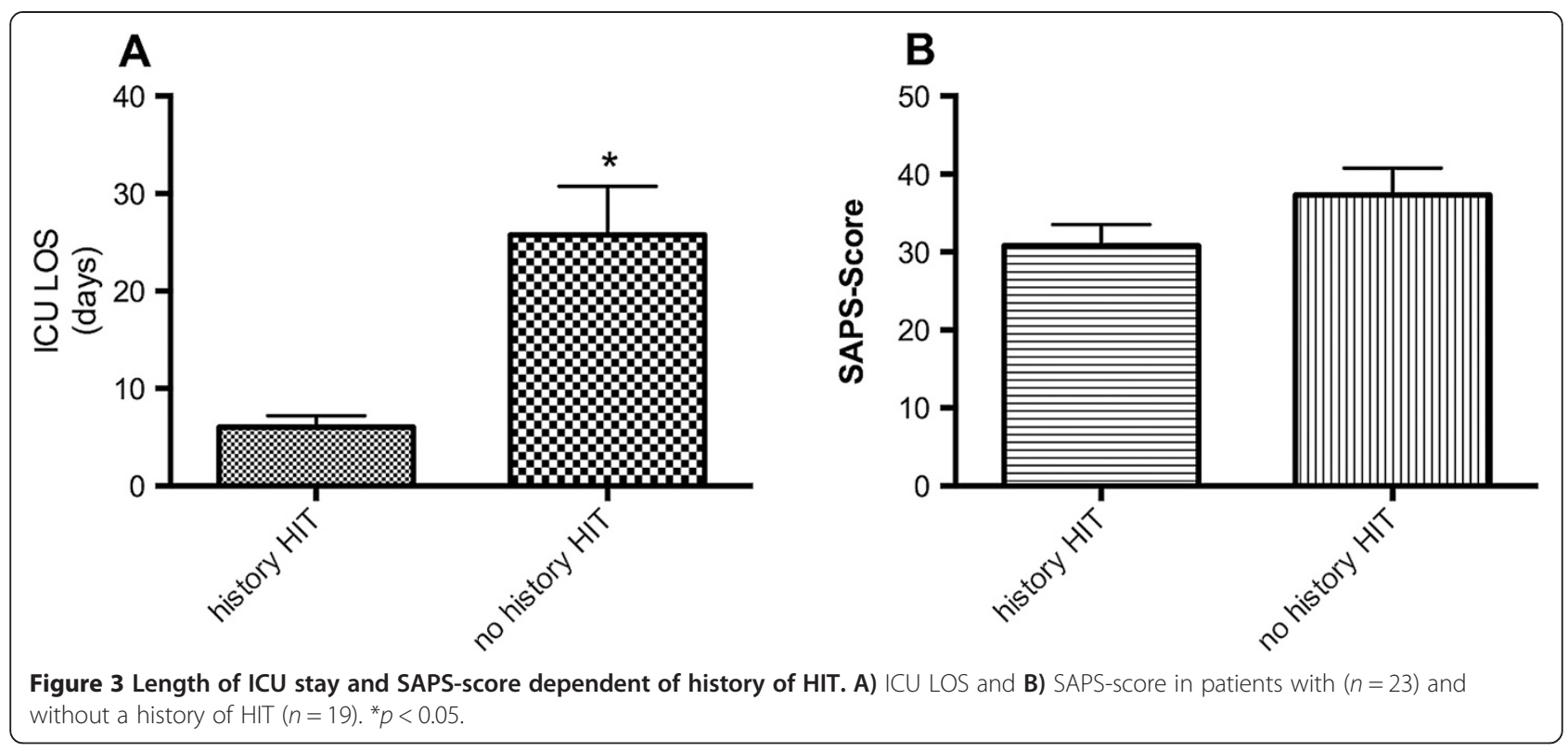

Considering these dosages and a wholesale price of 2.17 EUR for 25.000 IE heparin compared to 188.12 EUR for $250 \mathrm{mg}$ (37.6 EUR per $50 \mathrm{mg}$ ) argatroban, the costs per day are about 17 times higher for argatroban administration than they are for heparin. In patients with no history of HIT, thus receiving heparin, transfusion requirements were higher than for patient with a history of HIT before initiation of argatroban therapy $(4.4 \pm 2.8$ PRBC, $n=14$ vs. $9.9 \pm 3.6 \mathrm{PRBC}, n=18)$. Considering a PRBC unit price of 90 EUR, total costs for PRBC of 900 EUR for patients with no history of HIT under heparin versus 450 EUR for patients with a history of HIT have to be calculated. Patients had a mean ICU LOS of $7.5 \pm 1.3$ days before HIT suspicion was made. Assuming that these patients would have

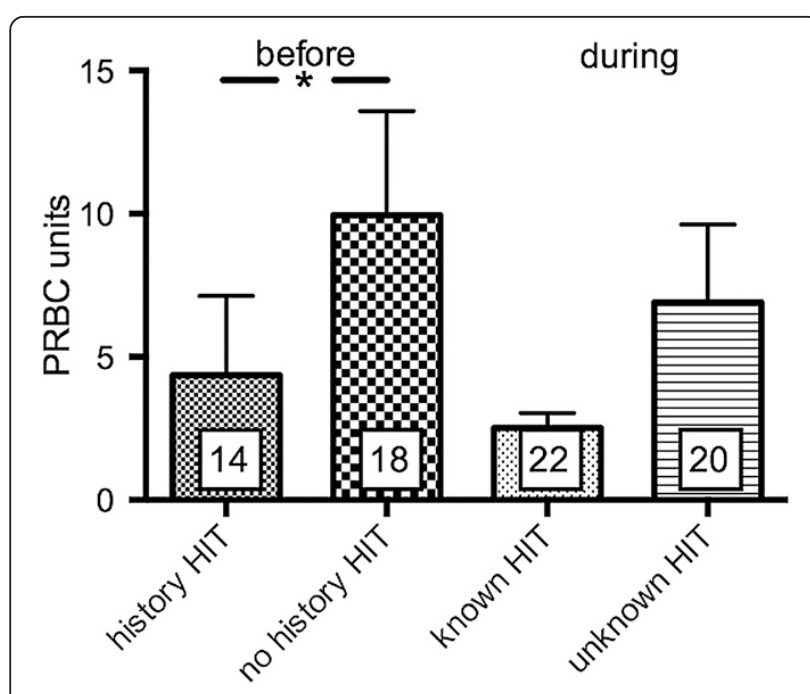

Figure 4 PRBC before and during argatroban therapy in patients with and without a history of HIT. ${ }^{*} p<0.05$. received argatroban in the first place instead of heparin, we estimate a total sum of 283.88 EUR for argatroban with a daily cost calculation of $37.60 \mathrm{EUR} / \mathrm{d}$ for argatroban multiplied with 7.5 days ICU LOS. This should be compared to 450 EUR additional costs for PRBC transfusions during heparin administration. Additional costs for laboratory testing (PF4 ELISA = 27.98 EUR, HIPAA = 52.46 EUR) would add up to a total of 364.32 EUR (Table 2).

Furthermore, patients with a history of HIT had a significant shorter ICU LOS. Assuming average costs of 1050.00 EUR per ICU day, total costs of ICU care in patients with a history of HIT would be 6 days $\times 1050$ $E U R=6300$ EUR versus 26 days $\times 1050$ EUR $=27300$ EUR in patients with no history of HIT, respectively

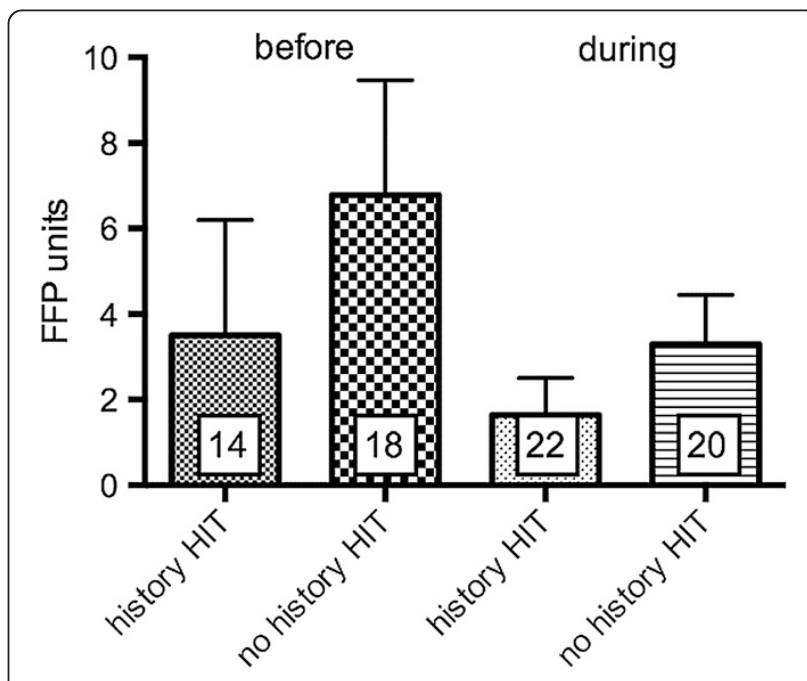

Figure 5 Fresh frozen plasma (FFP) units consumption (mean \pm SEM) before and during argatroban therapy in patients with known and unknown HIT. $p<0.05$. 


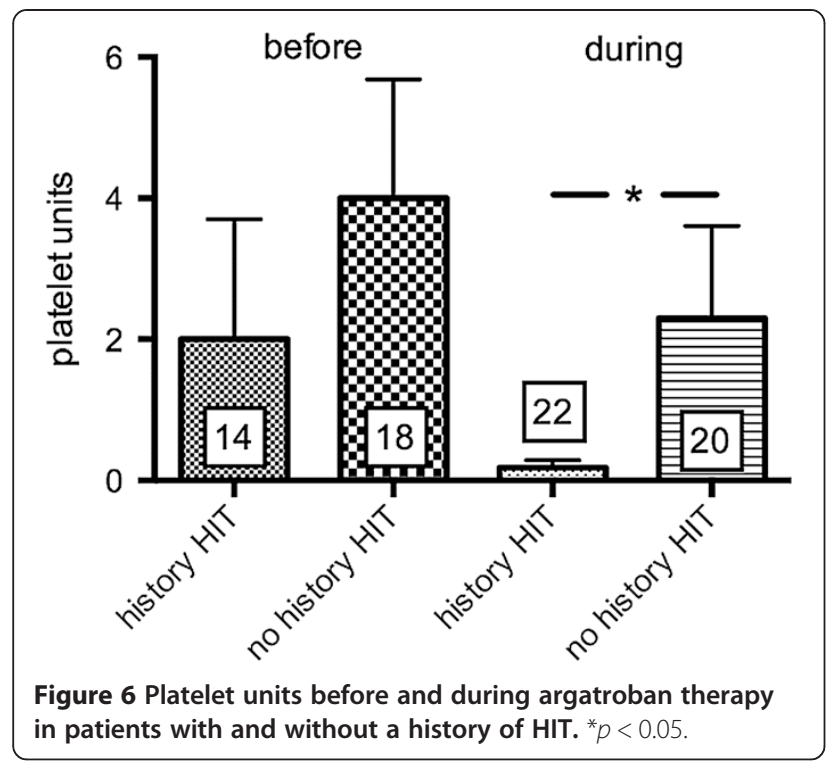

resulting in a $77 \%$ cost reduction for patients with a history of HIT (Table 2).

\section{Discussion}

The primary aims of this study were to investigate the safe dosage of argatroban in critically ill patients, thromboembolic complications and use of blood components. Furthermore, we analyzed the impact of a history of HIT on ICU LOS and the need for blood transfusions and the resulting economic consequences of argatroban versus heparin administration.

One striking finding of our study was that in our investigated population patients with a history of HIT had

Table 2 Comparison of costs for heparin versus argatroban

\begin{tabular}{lll}
\hline & Heparin & Argatroban \\
\hline Mean dosage/ICU day & 21744 IE & $49.6 \mathrm{mg}$ \\
Costs/ICU day & 2.17 EUR & $37.60 \mathrm{EUR}$ \\
$\begin{array}{l}\text { Total costs before HIT } \\
\text { (mean ICU LOS before HIT }\end{array}$ & $16.40 \mathrm{EUR}$ & $283.90 \mathrm{EUR}$ \\
$\begin{array}{l}\text { suspicion = 7.5 days) } \\
\text { Costs for HIT diagnostics }\end{array}$ & \\
& & 27.98 EUR (PF4 ELISA) \\
$\begin{array}{l}\text { PRBC transfusion before } \\
\text { HIT suspicion }\end{array}$ & 9.9 units & 4.4 units \\
$\begin{array}{l}\text { PRBC total costs before } \\
\text { HIT (unit costs = 90 EUR) }\end{array}$ & 900.00 EUR & 450.00 EUR \\
$\begin{array}{l}\text { Total costs for anticoagulation } \\
\text { and transfusions }\end{array}$ & 916.40 EUR & 814.34 EUR \\
ICU LOS & 26 & 6 \\
$\begin{array}{l}\text { Total costs of ICU stay } \\
\text { (1050EUR/day) }\end{array}$ & 27.000 .00 EUR & 6.300 .00 EUR \\
\hline
\end{tabular}

An ICU LOS of 7.5 days and increased RBC transfusion with heparin anticoagulation were assumed. For further details see text. a much shorter ICU LOS when compared to patients with no history of HIT, regardless of SAPS score. The reason for a longer ICU LOS remains unclear. Patients with no history of HIT develop thrombocytopenia during their ICU stay because heparin is usually administered perioperatively. Thrombocytopenia itself is not a reason for an extended ICU stay. However, once suspicion of HIT arises, patients are closely monitored for thromboembolic events and argatroban therapy, which can be best established in the ICU. Red blood cell transfusion need was lower in patients with a history of HIT before anticoagulation with argatroban compared with patients with no history of HIT. There was also a difference for FFP and platelet transfusion, but the differences did not reach statistical significance. Platelet transfusions were less frequent in patient with a history of HIT during argatroban therapy. These findings imply that patients with no history of HIT are more prone to bleeding and have greater transfusion needs due to inadequate anticoagulation with heparin and thrombocytopenia. A recent study by Williamson et al. demonstrated that critically ill patients with thrombocytopenia have a higher risk of bleeding with subsequent transfusion need and increased ICU and hospital mortality [21]. The reported incidence of HIT is $0.3 \%$ in perioperative patients and associated with a $50 \%$ increased mortality [22]. Clinical development and suspicion of HIT, which is based mainly on significant thrombocytopenia, is usually established over the course of a couple of days. Once HIT is suspected, argatroban therapy is initiated. It has to be taken into consideration that platelet count may further decrease and thromboembolic complications may have already developed [5]. This may also affect transfusion needs during the remaining course of the ICU stay. In contrast, early screening of patients for HIT with a PF4 ELISA on admission to the ICU leads to overdiagnosis of HIT since it remains a clinical diagnosis [9]. Although a safety profile of argatroban in critically ill patients still needs to be established, bleeding complications are also common in patients on heparin [23]. Considering the fatality of thromboembolic complications due to HIT, general use of argatroban could be favorable. Furthermore, laboratory screening tests for HIT would be redundant if agratroban were be used in the first place.

Our retrospective analysis confirmed the current practice that the dosage should not be higher than $0.5 \mathrm{mcg} /$ $\mathrm{kg} / \mathrm{min}$ in critically ill patients, as recommended by the manufacturer. Moreover, a dosage below $0.4 \mathrm{mcg} / \mathrm{kg} / \mathrm{min}$ can be considered safe in terms of thromboembolic events. Link et al. postulated a dosage calculation for argatroban in critically ill patients needing continuous renal replacement therapy using ICU scores such as APACHEII and SAPSII. A dose range of $0.5-1.2 \mathrm{mcg} / \mathrm{kg} / \mathrm{min}$ would correspond to SAPS scores 30-52 according to a predicted dosage calculation [13]. In our patient population 
we did not find a correlation between argatroban dosage and SAPS scores. Maintenance dosage had to be slightly increased in SAPS $<30$ to achieve aPTT with 1.5 to 3 -fold of baseline range. A SAPS score of 31 is consistent with severe illness and correlates with a predicted mortality of $11.7 \%[24,25]$. In SAPS $>30$ critically ill patients in this study population, maintenance dosage was unchanged when compared to initial dosage. In the past, studies in critically ill patients have defined an argatroban dose range of lower than $0.5 \mathrm{mcg} / \mathrm{kg} / \mathrm{min}[16,26,27]$. These low dosages are not only safer for patients in terms of bleeding complications but are also more cost-effective for both blood component and argatroban consumption.

For economic considerations and cost calculations, patients with no history of HIT who received heparin before HIT suspicion was raised served as a comparison group. Although costs of argatroban administration were 17 times higher per day compared to heparin administration, these costs would be compensated by a reduced need for transfusions if argatroban was used as a first line anticoagulant in patients with no history of HIT. Interestingly, patients with no history of HIT had a significantly longer ICU LOS, which ultimately accounts for higher total costs for intensive care. Considering the devastating thromboembolic complications in 30\% of cases associated with HIT, mortality rates up to $20 \%$ [28], an average cost-of-illness for confirmed HIT with thrombosis of 34155 Canadian Dollars $(=22432$ EUR/ 30847 USD in 2014) [29] and an incidence of up to 5\% [30], alternative anticoagulants such as argatroban could have a better economic profile than heparin itself, despite argatroban's higher market price.

As with all anticoagulants, major bleeding is one of the most common adverse events of argatroban therapy, but compared to a historical control bleeding complications are not increased [18,31]. Hepatic dysfunction and critical illness warrant dose adjustment as demonstrated by previous studies [16,27]. Doepker and coworkers identified argatroban-associated bleeding risk factors such as bilirubin $>3 \mathrm{mg} / \mathrm{dL}$, platelets $<70 \mathrm{~K} / \mathrm{mcL}$ and initial dosing body weight $>90 \mathrm{~kg}$ [18]. Starting dose ranges vary between $0.2 \mathrm{mcg} / \mathrm{kg} / \mathrm{min}$ in Beiderlinden's study and $0.56 \mathrm{mcg} / \mathrm{kg} / \mathrm{min}$ in Doepker's study. Kiser et al. investigated a fixed dosage adjustment protocol to achieve a target aPTT. Although, their data suggest that $78 \%$ of their patient population was located in the ICU and had a dose regimen based on organ dysfunction, the mean initial dosage for argatroban was $1.5 \mathrm{mcg} / \mathrm{kg} / \mathrm{min}$ and 1.3 $\mathrm{mcg} / \mathrm{kg} / \mathrm{min}$ for maintenance [32]. In the present retrospective study, we analyzed the safety of the dose ranges of argatroban administered to critically ill patients by assessing the need of transfusions before and during argatroban therapy. The overall need for blood component transfusions during argatroban therapy during ICU stay was not increased when compared with transfusion need before initiation of argatroban. However, argatroban therapy was temporarily discontinued when a major bleeding event occurred, during diagnostic and interventional procedures and with a supratherapeutic aPTT. While aPTT is the anticoagulation monitoring of choice for argatroban, incorporation in the daily ICU routine for HIT patients may be challenging due to multiple interruptions of therapy due to diagnostic or interventional procedures. Rotational thrombelastometry (ROTEM) evaluated in vitro shows a strong correlation between clotting time and aPTT. It could be a potential alternative for bedside monitoring of argatroban therapy [33]. However, major bleeding during argatroban therapy may still occur despite low dose regimen. Specifically, hepatic dysfunction (Child-Pugh class B) warrants dose reduction, since argatroban is metabolized in the liver by hydroxylation and aromatization of the 3-methyltetrahydroxyquinoline ring. In our study population, seven patients were identified with moderately elevated serum aminotransferases. Argatroban initial and maintenance dosage were below the manufacturer's recommended dosage of $0.5 \mathrm{mcg} / \mathrm{kg} / \mathrm{min}$ and did not differ when compared with all other study patients. Furthermore, the number of blood transfusions was not increased. However, argatroban therapy in patients with elevated aminotransferases had to be interrupted more often and for longer time intervals compared to all other patients. No thromboembolic complications were encountered in our patient population.

This study has some limitations. A heterogeneous surgical population was investigated in this retrospective analysis. Thus, bleeding risk factors are difficult to evaluate and argatroban therapy could have varying effects on overall bleeding. APTT and interruption of argatroban may not reflect a direct anticoagulatory effect of argatroban. Minimum aPTT range to prevent thrombembolic complications under argatroban was not evaluated. Furthermore, this study lacks a historical control group due to heterogeneity of the investigated patients. However, it was not the aim of this study to directly compare argatroban with heparin in terms of bleeding risk. We compared parameters before and after argatroban in patients who had no history of HIT, thus receiving heparin before HIT was suspected. The transfusion requirement data was not corrected for the time before initiation of argatroban. It has to be considered that this comparison group had a higher risk of bleeding with respect to HIT while heparin was still administered requiring more PRBC transfusions.

\section{Conclusion}

Low dosage of argatroban for critically ill patients is safe with respect to thromboembolic complications. Clinical monitoring of bleeding in the surgical population remains the most important parameter for adequate 
argatroban dosing while close aPTT monitoring remains a challenge for the daily ICU routine. Our data suggests that patients with new onset of HIT have an overall longer ICU stay and higher risk of bleeding. Cost calculations favor the use of argatroban in patients with no history of HIT necessitating laboratory screening. This should be investigated further.

\section{Competing interests}

The authors declare that they have no competing interests.

\section{Authors' contributions}

SK analyzed the data and drafted the manuscript. NT collected, analyzed the data and revised the manuscript, JCS analyzed the data and revised the manuscript, OB analyzed the data and revised the manuscript, MW helped to design the study and revised the manuscript, IG helped to design the study and revised the manuscript, $\mathrm{AH}$ designed the study and revised the manuscript, GB designed the study, analyzed the data, helped to draft and revised the manuscript. All authors read and approved the final manuscript.

\section{Acknowledgments}

Assistance with the study: Catherine Widmann edited the manuscript for English language.

\section{Financial support and sponsorship}

This work was supported by the Department of Anesthesiology and Intensive Care Medicine, University Hospital Bonn, Germany.

Received: 4 August 2014 Accepted: 18 January 2015

Published online: 07 February 2015

\section{References}

1. Visentin GP, Moghaddam M, Beery SE, McFarland JG, Aster RH. Heparin is not required for detection of antibodies associated with heparin-induced thrombocytopenia/thrombosis. J Lab Clin Med. 2001;138:22-31.

2. Greinacher A, Ittermann T, Bagemuhl J, Althaus K, Furll B, Selleng S, et al. Heparin-induced thrombocytopenia: towards standardization of platelet factor 4/heparin antigen tests. J Thromb Haemost. 2010;8:2025-31.

3. Lo GK, Juhl D, Warkentin TE, Sigouin CS, Eichler P, Greinacher A. Evaluation of pretest clinical score ( 4 T"s) for the diagnosis of heparin-induced thrombocytopenia in two clinical settings. J Thromb Haemost. 2006;4:759-65.

4. Cuker A, Gimotty PA, Crowther MA, Warkentin TE. Predictive value of the 4Ts scoring system for heparin-induced thrombocytopenia: a systematic review and meta-analysis. Blood. 2012;120:4160-7.

5. Greinacher A, Farner B, Kroll H, Kohlmann T, Warkentin TE, Eichler P. Clinical features of heparin-induced thrombocytopenia including risk factors for thrombosis. A retrospective analysis of 408 patients. Thromb Haemost. 2005:94:132-5.

6. Kitchens CS. Thrombocytopenia due to acute venous thromboembolism and its role in expanding the differential diagnosis of heparin-induced thrombocytopenia. Am J Hematol. 2004;76:69-73.

7. Warkentin TE, Greinacher A. Heparin-induced thrombocytopenia: recognition, treatment, and prevention: the Seventh ACCP Conference on Antithrombotic and Thrombolytic Therapy. Chest. 2004;126:311S-37.

8. Lee GM, Arepally GM. Heparin-induced thrombocytopenia. Hematology Am Soc Hematol Educ Program. 2013;2013:668-74.

9. Raschke RA, Curry SC, Warkentin TE, Gerkin RD. Improving clinical interpretation of the anti-platelet factor 4/heparin enzyme-linked immunosorbent assay for the diagnosis of heparin-induced thrombocytopenia through the use of receiver operating characteristic analysis, stratum-specific likelihood ratios, and Bayes theorem. Chest. 2013;144:1269-75.

10. Cuker A. Heparin-induced thrombocytopenia (HIT) in 2011: an epidemic of overdiagnosis. Thromb Haemost. 2011;106:993-4.

11. Fabris F, Luzzatto $G$, Soini $B$, Ramon $R$, Scandellari $R$, Randi ML, et al. Risk factors for thrombosis in patients with immune mediated heparin-induced thrombocytopenia. J Intern Med. 2002:252:149-54.

12. Berry C, Tcherniantchouk O, Ley EJ, Salim A, Mirocha J, Martin-Stone S, et al. Overdiagnosis of heparin-induced thrombocytopenia in surgical ICU patients. J Am Coll Surg. 2011;213:10-7. discussion 17.
13. Link A, Girndt M, Selejan S, Mathes A, Bohm M, Rensing H. Argatroban for anticoagulation in continuous renal replacement therapy. Crit Care Med. 2009;37:105-10.

14. Reichert MG, MacGregor DA, Kincaid EH, Dolinski SY. Excessive argatroban anticoagulation for heparin-induced thrombocytopenia. Ann Pharmacother. 2003;37:652-4.

15. Williamson DR, Boulanger I, Tardif M, Albert M, Gregoire G. Argatroban dosing in intensive care patients with acute renal failure and liver dysfunction. Pharmacotherapy. 2004;24:409-14.

16. Beiderlinden $M$, Treschan TA, Gorlinger K, Peters J. Argatroban anticoagulation in critically ill patients. Ann Pharmacother. 2007:41:749-54.

17. Cormack GM, Kaufman L. Severe heparin-induced thrombocytopenia: when the obvious is not obvious, a case report. J Med Case Reports. 2007;1:13.

18. Doepker B, Mount KL, Ryder $\amalg$, Gerlach AT, Murphy CV, Philips GS. Bleeding risk factors associated with argatroban therapy in the critically ill. J Thromb Thrombolysis. 2012;34:491-8.

19. Pratt DS, Kaplan MM. Evaluation of abnormal liver-enzyme results in asymptomatic patients. N Engl J Med. 2000;342:1266-71.

20. Ranieri VM, Rubenfeld GD, Thompson BT, Ferguson ND, Caldwell E, Fan $E_{\text {, }}$ et al. Acute respiratory distress syndrome: the Berlin Definition. JAMA. 2012;307:2526-33.

21. Williamson DR, Albert M, Heels-Ansdell D, Arnold DM, Lauzier F, Zarychanski R, et al. Thrombocytopenia in critically ill patients receiving thromboprophylaxis: frequency, risk factors, and outcomes. Chest. 2013;144:1207-15.

22. Seigerman M, Cavallaro P, Itagaki S, Chung I, Chikwe J. Incidence and outcomes of heparin-induced thrombocytopenia in patients undergoing cardiac surgery in North America: an analysis of the nationwide inpatient sample. J Cardiothorac Vasc Anesth. 2014;28(1):98-102.

23. De A, Roy P, Garg VK, Pandey NK. Low-molecular-weight heparin and unfractionated heparin in prophylaxis against deep vein thrombosis in critically ill patients undergoing major surgery. Blood Coagul Fibrinolysis. 2010;21:57-61.

24. Le Gall JR, Lemeshow S, Saulnier F. A new Simplified Acute Physiology Score (SAPS II) based on a European/North American multicenter study. JAMA. 1993:270:2957-63.

25. Le Gall JR, Neumann A, Hemery F, Bleriot JP, Fulgencio JP, Garrigues B, et al. Mortality prediction using SAPS I: an update for French intensive care units. Crit Care. 2005;9:R645-52.

26. Kodityal S, Nguyen PH, Kodityal A, Sherer J, Hursting MJ, Rice L. Argatroban for suspected heparin-induced thrombocytopenia: contemporary experience at a large teaching hospital. J Intensive Care Med. 2006;21:86-92.

27. Saugel B, Phillip V, Moessmer G, Schmid RM, Huber W. Argatroban therapy for heparin-induced thrombocytopenia in ICU patients with multiple organ dysfunction syndrome: a retrospective study. Crit Care. 2010;14:R90.

28. Fabris F, Luzzatto G, Stefani PM, Girolami B, Cella G, Girolami A. Heparin-induced thrombocytopenia. Haematologica. 2000;85:72-81.

29. Nanwa N, Mittmann N, Knowles S, Bucci C, Selby R, Shear N. The direct medical costs associated with suspected heparin-induced thrombocytopenia. Pharmacoeconomics. 2011;29:511-20.

30. Greinacher A, Warkentin TE. Recognition, treatment, and prevention of heparin-induced thrombocytopenia: review and update. Thromb Res. 2006:118:165-76.

31. Gray A, Wallis DE, Hursting MJ, Katz E, Lewis BE. Argatroban therapy for heparin-induced thrombocytopenia in acutely ill patients. Clin Appl Thromb Hemost. 2007;13:353-61.

32. Kiser TH, Mann AM, Trujillo TC, Hassell KL. Evaluation of empiric versus nomogram-based direct thrombin inhibitor management in patients with suspected heparin-induced thrombocytopenia. Am J Hematol. 2011;86:267-72.

33. Engstrom $M$, Rundgren $M$, Schott $U$. An evaluation of monitoring possibilities of argatroban using rotational thromboelastometry and activated partial thromboplastin time. Acta Anaesthesiol Scand. 2010;54:86-91. 